\title{
Study on the Optimum Extraction Conditions and Antibacterial Activities in Vitro of Total Flavonoids from Grape Leaves
}

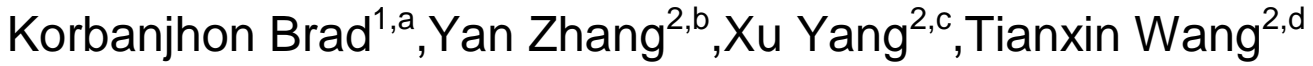 \\ ${ }^{1}$ College of Chemistry and Environmental Sciences, Yili Normal University,Yining, 835000, China, \\ ${ }^{2}$ Key Laboratory of Food Nutrition and Safety (Tianjin University of Science and Technology), \\ Ministry of Education, Tianjin 300457, China \\ akorbanjhon@126.com, ${ }^{\mathrm{b}} \mathrm{cpzyyan@126.com}$, \\ cxy1009@tust.edu.cn, , ${ }^{\mathrm{c} w t x w a n g @ t u s t . e d u . c n}$
}

Key words:grape leaves, total flavonoids, extracting technology, Antibacterial Activities

Abstract. The optimum extraction process of total flavonoids from grape leaves and their antibacterial activities in vitro were investigated. Total flavonoids were extracted by Soxhlet extraction. The content of total flavonoids in grape leaves was determined with aluminum nitrate chromogenic Spectrophotometry. The antibacterial activity was determined by K-B and agar diffusion method. The antibacterial test was done respectively by Escherichia coli, Staphylococcus aureus, Candida albicans, Bacillus subtilis, and choosing Staphylococcus aureus as the indicator organisms for the most obvious antibacterial effect. The single-factor test and orthogonal test taken the extraction rate of total flavonoids and the diameter of inhibition zone as index.Linear regression with concentration and absorbance of rutin standard solution, the regression equation was $\mathrm{Y}=$ $0.3295 \mathrm{X}+0.0012(\mathrm{R} 2=0.995)$, and the linear range was $0.4 \sim 2.0 \mathrm{mg} / \mathrm{mL}$. The content and extraction rate of total flavonoids in grape leaves were calculated according to the regression equation. Magnitude order of the effect of the four factors on the extraction rate and the antibacterial activities of total flavonoids in grape leaves was :extraction temperature> the concentration of ethanol> reflux time> ratio of material to liquid. The optimum extraction conditions were determined as follows: the concentration of ethanol (90\%), ratio of material to liquid (1:40), extraction temperature $\left(80^{\circ} \mathrm{C}\right)$, the extraction time $(3.5 \mathrm{~h})$. The average extraction rate of total flavonoids in grape leaves under the optimum extraction conditions was 7.16\%.Total flavonoids in grape leaves had a significant antibacterial effect on Staphylococcus aureus and Escherichia coli, and the effect on Staphylococcus aureus was the most obvious, but on Bacillus subtilis was not very remarkble.

\section{Introduction}

Grape (Vitu Vinifera L.), one of the world's oldest plant, is the Vitaceae vitis deciduous vines. Grapes are grown in most parts of China, especially in Xinjiang [1,2]. Grape leaves are used as Chinese traditional medicine, curing vomiting, edema, urination, red eyes and other symptoms. According to literatures, grape leaves contain abundant flavonoids with high physiological activity ,and organic acid, tannins, oligomeric stilbenes ,etc. Grape leaves are mostly used as cooking materials in Turkey, Greece and the Middle East area, and one of the most famous dishes is domasi , a dish with rice and meat in grape leaves [2-5]. The grape resources are rich in China, but a lot of grape leaves have not yet been fully developed and used.

Flavonoids, distributed widely in nature, often exist with vitamin $\mathrm{C}$, and belong to the secondary plant metabolites. Flavonoids are a group of natural pigment ,existing in vegetables, fruits, flowers and 
grains, named flavonoids because most of them are yellow . Researches show that flavonoids have many biological activities such as antioxidant, antibacterial, antiviral, maintenance cardiovascular system, anti-cancer, etc. In recent years, with the research methods and technology continue to improve, many new species and physiological function of flavonoids have been discovered, especially the anti free radical and anti-cancer effect, making the research of bioflavonoids have entered a new stage, setting off the upsurge of research, development and utilization of bioflavonoids. Flavonoids has broad application prospects in fields of food and medicine[6]. In this paper, the total flavonoids in grape leaves were extracted using ethanol as the extraction agent, and the optimum extraction conditions were found by orthogonal test based on single-factor test , and the antibacterial activities of the total flavonoids in grape leaves in vitro were determined .It will provide scientific basis for the extraction and utilization of total flavonoids in grape leaves .

\section{Experimental}

\section{Materials and equipment}

Grape leaves collected in September 18, 2013 from kale township of Turpan City, dried naturally,then drying on the electric heating temperature drying box at the temperature of $40^{\circ} \mathrm{C}$ to avoid light, crushing, stored at $4{ }^{\circ} \mathrm{C}$. The collected material was authenticated by Prof. Korbanjhon Brad, Key Laboratory at Universities of Education Department of Xinjiang Uygur Autonomous Region, Yili Normal University. Staphylococcus aureus, Bacillus subtilis, Escherichia coli, Canidia Albicans were purchased from Beijing Lai Yiu biological science and Technology Co Ltd.Beef extract peptone agar medium, LB medium, potato culture medium were purchased from Hangzhou microbial reagent limited company. rutin standard (98\%) was purchased from Sinopharm Chemical Reagent Co. Ltd..Agar agar powder, peptone were purchased from Tianjin city Yingbo biochemical reagent Co. Ltd.. Beef extract was purchased from Beijing aoboxing biotechnology limited liability company. Other chemicals were of analytical grade and used as received.

\section{Optimization of the extraction technology of total flavonoids from grape leaves (Single-factor} test and Orthogonal test)

The grape leaves were collected a number of copies (each about $2 \mathrm{~g}$ ), to $250 \mathrm{~mL}$ Soxhlet extractor, then treated with different concentrations of ethanol as extracting agent. The effects of ethanol concentration, extraction time, extraction temperature and solid - liquid ratio on extraction rate and antibacterial activities of total flavonoids in grape leaves were investigated respectively, taking the content of total flavonoids and inhibition zone diameter as index.

Four most influential factors were selected based on single-factor test, and an L9(34) orthogonal test were designed. The extract absorbance values were determined, and the extraction rate of flavonoids was calculated, then magnitude order of the effect of the four factors on extraction rate and antibacterial test of flavonoids were analysed, to determine the optimal conditions of extraction.

\section{Determination of the Content of Flavonoids}

The content of flavonoids was measured as rutin quivalents from a rutin standard curve. One ml of the sample extract was transferred to a test tube, the solution was redissolved in $30 \%$ ethanol to 12.5 $\mathrm{ml}$ and $0.7 \mathrm{ml}$ of $5 \% \mathrm{NaNO}_{2}$ reagent was added. After an incubation period of $5 \mathrm{~min}, 0.7 \mathrm{~mL}$ of $\mathrm{Al}\left(\mathrm{NO}_{3}\right)_{3}$ was added, mixed well and kept for $6 \mathrm{~min}$ at room temperature,5ml of $1 \mathrm{M} \mathrm{NaOH}$ reagent was added. The solution was redissolved in $30 \%$ ethanol to $25 \mathrm{ml}$. The above solution was incubated for $10 \mathrm{~min}$, and then the absorbance was readed at $510 \mathrm{~nm}$ using a spectrophotometer.

\section{Determination of the content of total flavonoids in samples}

The extract was transferred to a $10 \mathrm{ml}$ volumetric flask, then operated according to The standard curve drawing, the extract absorbance at a wavelength of $510 \mathrm{~nm}$ was determined by 
UV.According to the standard curve, the content and extraction rate of total flavonoids in sample was calculated using Eq.1.

$$
\mathrm{E}=\frac{\mathrm{YaV}}{\mathrm{W}}
$$

Eq. 1

Where Y represents concentration of flavonoids $(\mathrm{mg} / \mathrm{mL})$; V represents crude extract volume $(\mathrm{mL})$; W represents samples quality (g); a represents dilution factor.

Determination of antibacterial activities in vitro (Bacteriostatic method: Inhibition of bacteria (filter paper method) and Inhibition of fungoid (filter paper method))

$10 \mu \mathrm{L}$ cultured bacteria suspension was added to a Petri dish with beef extract peptone solid culture medium under sterile conditions, coated evenly with sterile triangular glass. According to the aseptic operation requirement ,the pre dried and sterilized filter paper flats( $6.0 \mathrm{~mm}$ ) were dip in grape leaves ethanol extract, then paste on bacteria containing medium, each plate 3 filter paper flats ,spaced a certain distance and triangular shaped arrangement, and one of the three was blank control .The tests were repeated 3 times. The prepared plates were put into the incubator at $37^{\circ} \mathrm{C}$, for $24 \mathrm{~h}$,then observed and the inhibition zone diameters were measured.

under sterile conditions, $10 \mu \mathrm{L}$ cultured fungoid suspension absorbs was added to the culture dish with PDA culture medium, coated evenly with sterile triangular glass. According to the aseptic operation requirement, the pre dried and sterilized filter paper flats $(6.0 \mathrm{~mm})$ were dip in grape leaves ethanol extract, then paste on bacteria containing medium, each plate 3 filter paper flats ,spaced a certain distance and triangular shaped arrangement, one of the three was blank control . The tests were repeated 3 times. The prepared plates were put into the incubator at $24^{\circ} \mathrm{C}$,for $24 \mathrm{~h}$, then observed and the inhibition zone diameters were measured.

\section{Results}

\section{Standard curve drawing}

\begin{tabular}{cccccc}
\multicolumn{5}{l}{ Tab. 1 The relationship between concentration and absorbance of rutin standard solution } \\
\hline $\begin{array}{c}\text { Concentration of Rutin } \\
(\mathrm{mg} / \mathrm{mL})\end{array}$ & 0.40 & 0.80 & 1.20 & 1.60 & 2.00 \\
\hline Absorbance & 0.1374 & 0.2575 & 0.3988 & 0.5275 & 0.6613 \\
\hline
\end{tabular}

The linear regression analysis on absorbance of rutin to the concentration of standard solution, the regression equation was: $\mathrm{Y}=0.3295 \mathrm{X}+0.0012(\mathrm{R} 2=0.995)$. The results showed that, the standard solution of rutin concentration in the range of $0.4 \sim 2.0 \mathrm{mg} / \mathrm{mL}$ showed a good linear relationship.

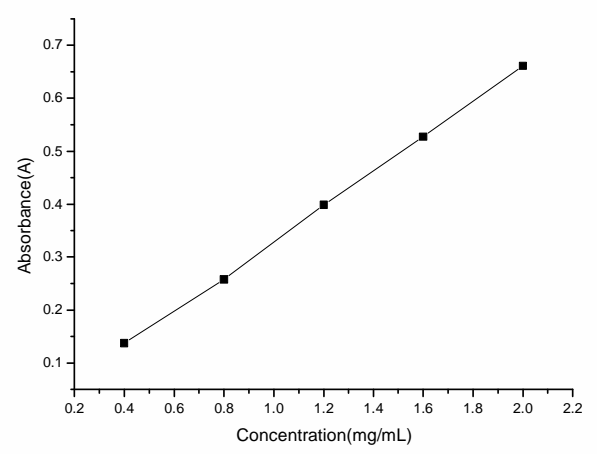

Fig. 1 standard curve of rutin 


\section{The results of single-factor test:Effect of concentration of ethanol on extraction rate and antibacterial effect of total flavonoids}

Grape leaves powder was weighed accurately (each $2 \mathrm{~g}$ ), placed in a Soxhlet extractor. According to the solid-liquid ratio of 1:40,ethanol solution was added , the concentrations were $45 \%, 60 \%, 75 \%$, $90 \%$, respectively. The extraction lasted for $3.5 \mathrm{~h}$, at $70^{\circ} \mathrm{C}$. Some of the extract was used to determine the absorbance by UV.The mass and extraction rate of total flavonoids were calculated according to the regression equation(Tab.2). The remaining extract was vacuum concentrated through rotary evaporation ,the ethanol recovery. The dried extract dissolved in $5 \mathrm{ml}$ sterile water. The four kinds of extraction solution were used in the antibacterial test respectively . Shown in Fig.2.

Tab. 2 effect of ethanol concentration on the extraction rate of flavonids and its inhibition zone size

\begin{tabular}{cccc}
\hline $\begin{array}{c}\text { ethanol } \\
\text { concentration }(\%)\end{array}$ & obsorbance & $\begin{array}{c}\text { Extraction } \\
\text { rate }(\%)\end{array}$ & $\begin{array}{c}\text { inhibition } \\
\text { zone diameter } \\
(\mathrm{mm})\end{array}$ \\
\hline 45 & 0.252 & 5.67 & 7.27 \\
60 & 0.4906 & 11.14 & 12.20 \\
75 & 0.4921 & 11.17 & 12.93 \\
90 & 0.4454 & 10.11 & 9.40 \\
\hline
\end{tabular}

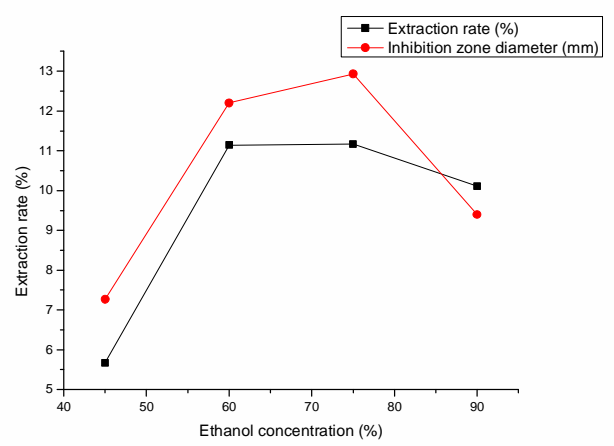

Fig. 2 The effect of ethanol concentration on extraction rate and the size of inhibition zone of total Flavonoids

We can see from Fig. 2, when the concentration of ethanol in the range of 45\%-75\%, the yield rate of flavonoids showed a rising trend with the increase of the concentration of solvent generally. When the concentration of ethanol increased to $75 \%$,the yield rate increased to a peak value, when more than $75 \%$, the yield rate declined. In the test it was found that as the concentration of ethanol increased, fat soluble substances dissolution increased, and the fat soluble substances had interference on the determination of absorbance, which made the measurement result high, and was not conducive to the further purification of flavonoids. It can be seen in Tab.2, the size of inhibition zone increased gradually with the increase of ethanol concentrations, the most obvious antibacterial effect of ethanol concentration was $75 \%$, then with the increase of the concentration of ethanol, inhibition zone gradually became smaller. The two charts taken together ,the amount of flavonoids affect the antibacterial activities of extract of grape leaves.

\section{The influence of solid-liquid ratio on the extraction rate of flavonoids and antibacterial effect}

Grape leaves powder was weighed accurately (each $2 \mathrm{~g}$ ), placed in a Soxhlet extractor. The solid-liquid ratios were 1:20、1:30、1:40、1:50 respectively, $75 \%$ ethanol solution was added. The extraction lasted for $3.5 \mathrm{~h}$, at $70^{\circ} \mathrm{C}$. Some of the extract was used to determined the absorbance by UV.The mass and extraction rate of total flavonoids were calculated according to the regression 
equation(Tab.3). The antibacterial effect on Staphyloccocus aureus of the four kinds of extraction solution was shown in Fig.3.

Tab. 3solid-liquid ratio effect on the extraction rate and inhibition zone size of total flavonoids

\begin{tabular}{cccc}
\hline $\begin{array}{c}\text { solid-liquid } \\
\text { ratio }\end{array}$ & absobance & $\begin{array}{c}\text { extraction rate } \\
(\%)\end{array}$ & $\begin{array}{c}\text { inhibition zone } \\
\text { size }(\mathrm{mm})\end{array}$ \\
\hline $1: 20$ & 0.3884 & 4.00 & 4.89 \\
$1: 30$ & 0.3487 & 5.80 & 7.12 \\
$1: 40$ & 0.2944 & 6.67 & 8.75 \\
$1: 50$ & 0.2244 & 6.44 & 8.53 \\
\hline
\end{tabular}

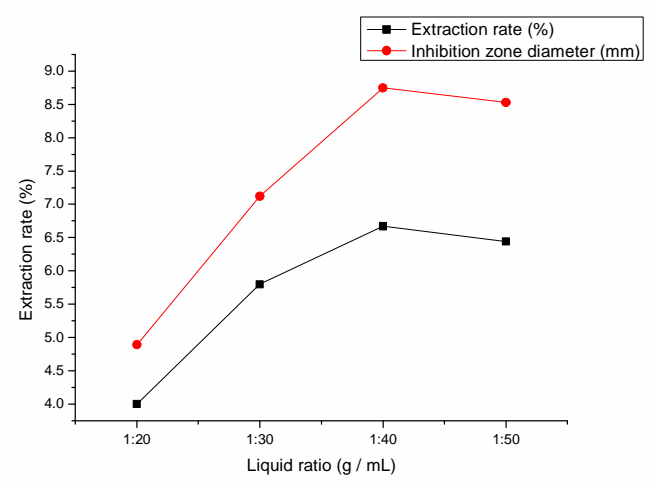

Fig. 3 effect of solid-liquid ratio on the rate of total flavonoids

We can see from Fig. 3, when the ratio of solid-liquid ratio in the range of 1:20 to 1:40, the yield rate of flavonoids showed a rising trend with the increase of the solid-liquid ratio generally, when the solid-liquid ratio increased to1:40,the yield rate increased to a peak value, when more than 1:40, the yield rate declined. The size of inhibition zone increased gradually with the increase of solid-liquid ratio, the most obvious antibacterial effect of solid-liquid ratio was 1:40(diameter of inhibition zone $8.75 \mathrm{~mm}$ ). It can be seen in Tab. 3 , when the solid-liquid ratio was 1:20,the content of flavonoids in the extract was few ,the antibacterial activities were also low ,it may be explained that when solid-liquid ratio was low, the solvent was not enough to dissolve the substance with antibacterial activities. When the solid-liquid ratio was $1: 30$ to $1: 50$, the average diameter of inhibition zone showed no significant difference. When the solid-liquid ratio was 1:40, the effect of extraction was good enough , the increase of the amount of solvent had no significant effect on the antibacterial activities. Taken the production cost in consideration, the best solid-liquid ratio was 1:40, and it was used in the single-factor test.

\section{The influence of extraction time on the extraction rate of flavonoids and antibacterial effect}

Grape leaves powder was weighed accurately (each $2 \mathrm{~g}$ ), placed in a Soxhlet extractor. The solid-liquid ratio was 1:40, and $75 \%$ ethanol solution was added .The extraction time was $1.5 \mathrm{~h}, 2.5 \mathrm{~h}, 3.5 \mathrm{~h}, 4.5 \mathrm{~h}$ respectively. Some of the extract was used to determine the absorbance by UV.The mass and extraction rate of total flavonoids were calculated according to the regression equation(Tab. 4). The antibacterial effect on Staphyloccocus aureus of the four kinds of extraction solution were shown in Fig. 4. 
Tab. 4 The influence of extraction time on the extraction rate and antibacterial effect of total flavonoids

\begin{tabular}{cccc}
\hline $\begin{array}{c}\text { extraction time } \\
(\mathrm{h})\end{array}$ & absorbance & $\begin{array}{c}\text { extraction rate } \\
(\%)\end{array}$ & $\begin{array}{c}\text { Inhibition } \\
\text { zone }(\mathrm{mm})\end{array}$ \\
\hline 1.5 & 0.3111 & 7.05 & 6.87 \\
2.5 & 0.3145 & 7.04 & 6.981 \\
3.5 & 0.3248 & 7.27 & 8.13 \\
4.5 & 0.2062 & 4.67 & 4.89 \\
\hline
\end{tabular}

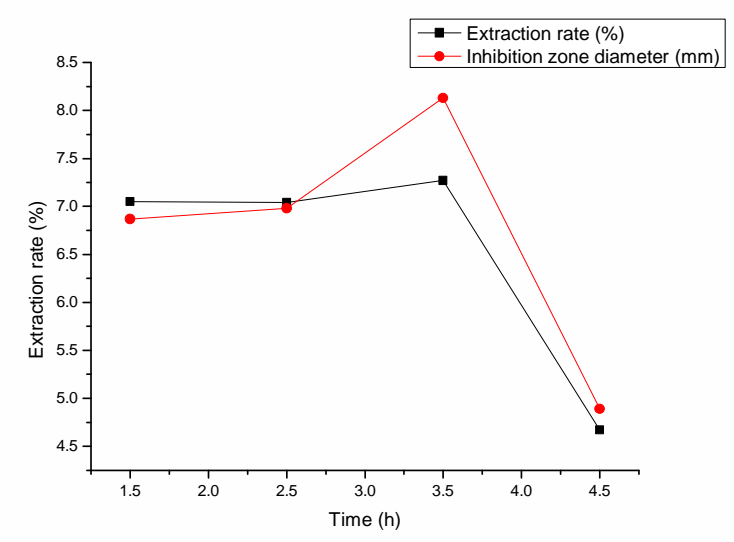

Fig. 4 effect of extraction time on the extraction rate of totalflavonoids

We can see from Fig. 4, when the extraction time in the range of $1.5 \mathrm{~h}$ to $3.5 \mathrm{~h}$, the yield rate of flavonoids showed no significant difference. $3.5 \mathrm{~h}$ was best for the extraction rate, then with the increase of time ,the content of total flavonoids declined, which may be explained that when the extraction time was too long, some ethanol evaporated, making the boiling point of the solvent increase and some flavonoids may be damaged. It can be seen in Tab.4, with the extraction time increased, antibacterial activities of the extract of grape leaves firstly increased and then decreased. $3.5 \mathrm{~h}$ was best for the antibacterial activities(average diameter $8.13 \mathrm{~mm}$ ). When extraction time was shorter than $3.5 \mathrm{~h}$, the extraction rate of flavonoids was too low, which may be explained that when the heating time was too short, the substance with antibacterial activities was not extracted enough , and when it was longer than $3.5 \mathrm{~h}$, some ethanol evaporated, making the boiling point of the solvent increase and some substance with antibacterial activities may be damaged, so the antibacterial effect decreased .

The influence of extraction temperature on the extraction rate and antibacterial effect of flavonoids

Grape leaves powder was weighed accurately (each $2 \mathrm{~g}$ ), placed in a Soxhlet extractor. The solid-liquid ratio was $1: 40$, and $75 \%$ ethanol solution was added The extraction temperature was $60^{\circ} \mathrm{C} 、 70^{\circ} \mathrm{C} 、 80^{\circ} \mathrm{C} 、 90^{\circ} \mathrm{C}$ respectively. The extraction time was $3.5 \mathrm{~h}$.Some of the extract was used to determined the absorbance by UV.The mass and extraction rate of total flavonoids were calculated according to the regression equation(Tab. 5). The antibacterial effect on Staphyloccocus aureus of the four kinds of extraction solution was shown in Fig. 5. 
Tab. 5 effect of extraction temperature on extraction rate and inhibition zone of flavonoids

\begin{tabular}{cccc}
\hline $\begin{array}{c}\text { extraction } \\
\text { temperature }\left({ }^{\circ} \mathrm{C}\right)\end{array}$ & absorbance & $\begin{array}{c}\text { extraction rate } \\
(\%)\end{array}$ & $\begin{array}{c}\text { inhibition zone } \\
\text { diameter }(\mathrm{mm})\end{array}$ \\
\hline 60 & 0.24 & 5.44 & 7.37 \\
70 & 0.303 & 6.78 & 8.89 \\
80 & 0.217 & 4.91 & 4.97 \\
90 & 0.367 & 8.33 & 5.59 \\
\hline
\end{tabular}

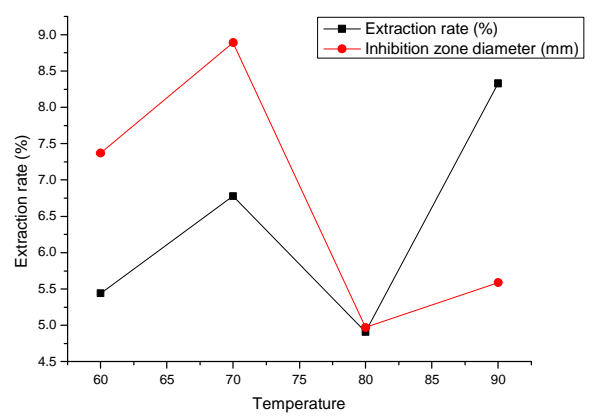

Fig. 5 effect of extraction temperature on extraction rate and inhibition zone of flavonoids

We can see from Tab.5, the yield rate of flavonoids varied significantly with the increase of extraction temperature . It may be explained that the solubility of flavonoids in ethanol increased with the increase of temperature, and at the same time, the viscosity of extracting solution decreased, and coefficient of diffusivity increased, which makes the rate of extract increased. But if the temperature was too high ,some active substance may be damaged , and the amount of foreign material in the extract may increase, which made later operations inconvenient. In addition, it made solvent loss and the cost increase. Considering various factors, $70^{\circ} \mathrm{Cwas}$ best for the antibacterial activities(average diameter of inhibition zone $8.89 \mathrm{~mm}$ ). When the temperature ranged from $60^{\circ} \mathrm{C}$ to $70^{\circ} \mathrm{C}$, with the increase of temperature ,the antibacterial activities increased .It may be explained that with the increase of temperature ,the extraction process was influenced not only by dissolution effect but also by thermal effect ,higher temperature made higher rate of extraction and better antibacterial effect. When temperature was higher than $70^{\circ} \mathrm{C}$, the antibacterial activities declined. It may be explained that when temperature was higher than $70^{\circ} \mathrm{C}$, some active substance may be damaged. When temperature ranged from $80^{\circ} \mathrm{C}$ to $90^{\circ} \mathrm{C}$, the effect of antibacterial increased with the increase of temperature .It may because other substance with antibacterial activities dissolved into the extract. So according to our results, $70^{\circ} \mathrm{Cwas}$ the best extraction temperature.

\section{The results of orthogonal test}

In the experiment, to comprehensively consider the process parameter of ethanol extraction, the concentration of ethanol, solid-liquid ratio, extraction time and extraction temperature four factors were chosen ,because of cross influence among various factors. According to the results of single-factor test, the $\mathrm{L}_{9}\left(3^{4}\right)$ orthogonal test was done to optimize the extraction conditions.

Tab. 6 levels of factors

\begin{tabular}{lllll}
\hline \multirow{2}{*}{ factor } & $\begin{array}{l}\text { concentration } \\
\text { of ethanol }\end{array}$ & $\begin{array}{l}\text { solid-liquid } \\
\text { ratio }\end{array}$ & $\begin{array}{l}\text { extraction } \\
\text { temperature }\end{array}$ & $\begin{array}{l}\text { extraction } \\
\text { time }\end{array}$ \\
& $\mathrm{A}(\%)$ & $\mathrm{B}(\mathrm{g} / \mathrm{mL})$ & $\mathrm{C}\left({ }^{\circ} \mathrm{C}\right)$ & $\mathrm{D}(\mathrm{h})$ \\
\hline level1 & 60 & $1: 20$ & 60 & $1.5 \mathrm{~h}$ \\
level 2 & 75 & $1: 30$ & 70 & $2.5 \mathrm{~h}$ \\
level 3 & 90 & $1: 40$ & 80 & $3.5 \mathrm{~h}$ \\
\hline
\end{tabular}


Tab. 7 result of orthogonal test

\begin{tabular}{lllllll}
\hline factor & $\begin{array}{l}\text { concentration } \\
\text { of ethanol }\end{array}$ & $\begin{array}{l}\text { extraction } \\
\text { temperature }\end{array}$ & $\begin{array}{l}\text { solid-liquid } \\
\text { ratio }\end{array}$ & $\begin{array}{l}\text { extraction } \\
\text { time }\end{array}$ & $\begin{array}{l}\text { extraction } \\
\text { rate }\end{array}$ & $\begin{array}{l}\text { inhibition } \\
\text { zone diameter } \\
(\mathrm{A})\end{array}$ \\
\hline level1 & 1 & 1 & 1 & $(\mathrm{C})$ & $(\mathrm{D})$ & $(\mathrm{mm})$ \\
level2 & 1 & 2 & 2 & 2 & 5.77 & 5.96 \\
level3 & 1 & 3 & 3 & 3 & 6.6 & 4.97 \\
level4 & 2 & 1 & 2 & 3 & 3.63 & 4.33 \\
level5 & 2 & 2 & 3 & 1 & 4.41 & 3.97 \\
level6 & 2 & 3 & 1 & 2 & 6.97 & 11.4 \\
level7 & 3 & 1 & 3 & 2 & 5.16 & 4.21 \\
level8 & 3 & 2 & 1 & 3 & 6.2 & 9.9972 \\
level9 & 3 & 3 & 2 & 1 & 6.71 & 6.89 \\
average1 & 4.79 & 3.59 & 5.05 & 4.37 & & \\
average2 & 5 & 5.46 & 5.37 & 5.97 & & \\
average3 & 6.02 & 6.76 & 5.39 & 5.48 & & \\
range & 1.23 & 3.17 & 0.34 & 1.11 & & \\
\hline
\end{tabular}

The experiment results showed: flavonoids were rich in grape leaves. When ethanol was used as extraction agent, the extraction temperature was the key factor affecting the extraction rate of flavonoids. Magnitude order of the effect of the four factors on the extraction rate of total flavonoids in grape leaves was: extraction temperature> the concentration of ethanol> reflux time> solid-liquid ratio. The optimum extraction conditions were determined as $\mathrm{A}_{3} \mathrm{~B}_{3} \mathrm{C}_{3} \mathrm{D}_{2}$ : the concentration of ethanol $(90 \%)$, extraction temperature $\left(80^{\circ} \mathrm{C}\right)$, solid-liquid ratio $(1: 40)$, extraction time(3.5h).

\section{Antibacterial test under the optimum conditions}

The orthogonal experiment determined the optimum conditions of extraction of grape leaves :ethanol concentration $(90 \%)$, extraction temperature $\left(80^{\circ} \mathrm{C}\right)$, solid-liquid ratio $(1: 40)$, extraction time( $3.5 \mathrm{~h})$, and under these conditions ,the ethanol extract of the grape leaves were extracted, and the average content of total flavonoids was $71.6 \mathrm{mg} / \mathrm{g}$.

Escherichia coli, Staphylococcus aureus, Bacillus subtilis, Candida albicans were used as test bacteria, using filter paper method and in strict accordance with the aseptic operation. The tests were repeated 3 times. The inhibition zone diameters of four kinds of bacteria were shown in Tab.8.

Tab. 8 comparison of antibacterial effect of total flavonoids extracted under optimum conditions

\begin{tabular}{ccccc}
\hline \multirow{2}{*}{ strains } & 1 & 1 & 3 & $\begin{array}{c}\text { Average } \\
\text { diameter }\end{array}$ \\
\cline { 2 - 5 } & & & \multicolumn{4}{c}{ Inhibition zone $(\mathrm{mm})$} \\
\hline staphylococcus & 11.89 & 12.3 & 11.74 & 11.98 \\
$\begin{array}{c}\text { aureus } \\
\text { Escherichia coli })\end{array}$ & 9.8 & 10.1 & 9.89 & 9.93 \\
Canidia Albicans & 5.97 & 6.36 & 6.57 & 6.3 \\
Bacillus subtilis & 4.3 & 3.9 & 3.87 & 4.1 \\
\hline
\end{tabular}




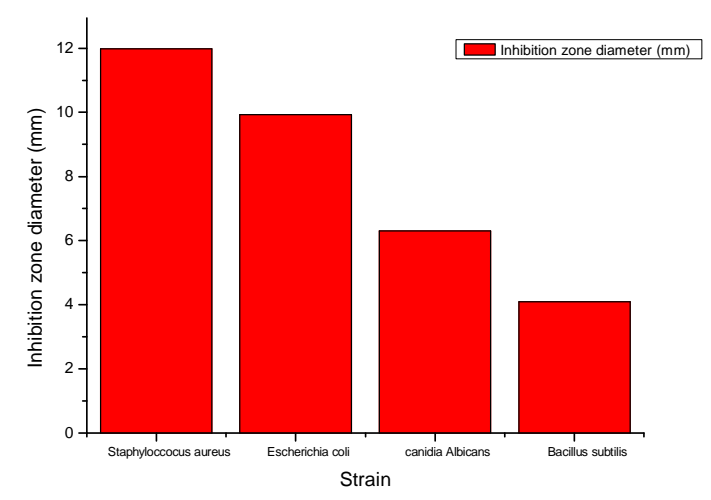

Fig. 6 comparison of antibacterial effect of total flavonoids extracted under optimum conditions

Under the optimum conditions of extraction, the antibacterial effects of ethanol extract of grape leaves on Staphylococcus aureus and Escherichia coli were remarkable, but on Bacillus subtilis was not very significant.

\section{Discussion}

There are many methods to extract natural products, but different extraction methods will have a certain impact on the experimental results. Soxhlet extraction method used in the experiment is an ideal way to extract flavonoids. Soxhlet extraction method has the following advantages: (1) low energy consumption: because the Soxhlet extraction method directly heats the extraction agent ,and the extraction agent selected always has a low boiling point, which fundamentally guarantees the rapid conduction and full use of energy. In addition, the extraction agent is recycled in. This not only reduces the amount of solvent, but also the operation time is shortened, then the energy consumption is greatly reduced; (2)The equipment is simple and easy to operate; (3) Soxhlet extraction method has the advantages of low cost, and the Soxhlet extractor has a small volume. In addition, it improves the purity of the extract of grape leaves.

In this experiment, total flavonoids extraction requires not only the extraction solvent having good solubility of flavonoids, but also having the ability to weaken the hydrogen bonding. Commonly used acetone-water solution reflux extraction can be used for phenolic hydroxyl and protein, polysaccharide and other effects. But because of toxic, volatile, residue acetone in product having harmful to the human body, and making the fat soluble components increase, acetone-water solution reflux extraction brings trouble to latter processing technology and production. There are also toxic in methanol extraction. After comprehensive consideration, this experiment selected ethanol solution as the extraction solvent. The solvent has the advantages such as high extraction rate, good selectivity and strong permeability, non-toxic, inexpensive, easy recovery and removal, no pollution to environment, etc.

Experiments results show that in the extraction of total flavonoids in grape leaves, ethanol concentration, solid-liquid ratio, temperature and time had different effects on the rate of total flavonoids. The extraction rate using $75 \%$ ethanol was high, which may indicate that it contains certain fat soluble flavonoids. When extraction temperature increased, inter molecular motion increased, and the content of flavonoids in refluxing extraction solution had a trend of increase. From the single-factor test we can see, extraction temperature and time should be appropriate, not too high or too low. When the temperature was too high or too long, the activity of antibacterial 
substances in grape leaves would decrease. Through setting different ethanol concentration gradient, the concentration about $75 \%$ was the best. When the concentration was less than $75 \%$, antibacterial substance in grape leaves was not easy to be extracted, but when the concentration was too high, the fat soluble substances dissolution increased, and had certain influence on the antimicrobial effect. In the selection of the solid- liquid ratio, too small solid-liquid ratio would cause the incomplete extraction, while too large would cause the waste of ethanol solvent.

\section{Conclusions}

The optimum extraction conditions of the total flavonoids from grape leaves were determined: the concentration of ethanol $(90 \%)$, solid-liquid ratio $(1: 40)$, extraction temperature $\left(80^{\circ} \mathrm{C}\right)$, the extraction time (3.5h).The total flavonoids from grape leaves had remarkable antibacterial effect on Staphylococcus aureus and Escherichia coli, and the biggest antibacterial activity on Staphylococcus aureus. But the antibacterial effect on Bacillus subtilis was not obvious. The order of antibacterial activities of total flavonoids from grape leaves on the four test organism was: Staphylococcus aureus, Escherichia coli, Candida albicans, Bacillus subtilis.

\section{Acknowledgements}

The Project Supported by the National Science Foundation of China (31401663), the Foundation of Tianjin University of Science and Technology, Institute for New Rural Development, P. R. China (No. XNC201511), and Youth Innovation Foundation of Tianjin University of Science and Technology (No. 20151G24).

\section{References}

[1] Z.Y. Zhang, Y. PENG: Biotech Vol.17(2007), p. 58

[2] M.N Bian, S.Z. Li, H.J. Bai, B.L. Liu,C. Li: Xinjiang Agr Sci Vol. 50(2013), p. 2037

[3] K. Brad, Y. Zhang, W. Liu: J Chem Soc Pakistan Vol. 35(2013), p. 1103

[4] L.L Sun, J. Kang, J.Y. Zou, H.H. Liu: Food Sci and Tech Vol. 39(2014), p.58

[5] Nilgün Göktürk Baydar, Gülcan Özkan, Samim Yaşar : Food Control Vol.18(2007), p.1131

[6] G.L. Zhang, Z. Wang, H.D: J Bio Vol. 22(2005), p. 4 